\title{
Room temperature operation of $n$-type Ge/ SiGe terahertz quantum cascade lasers predicted by non-equilibrium Green's functions
}

Cite as: Appl. Phys. Lett. 114, 111102 (2019); https://doi.org/10.1063/1.5082172

Submitted: 19 November 2018 • Accepted: 24 February 2019 • Published Online: 20 March 2019

(iD) Thomas Grange, (D) David Stark, (D) Giacomo Scalari, et al.
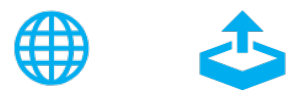

\section{ARTICLES YOU MAY BE INTERESTED IN}

Thermoelectrically cooled THz quantum cascade laser operating up to $210 \mathrm{~K}$ Applied Physics Letters 115, 010601 (2019); https://doi.org/10.1063/1.5110305

THz intersubband electroluminescence from n-type Ge/SiGe quantum cascade structures Applied Physics Letters 118, 101101 (2021); https://doi.org/10.1063/5.0041327

Modeling techniques for quantum cascade lasers

Applied Physics Reviews 1, 011307 (2014); https://doi.org/10.1063/1.4863665

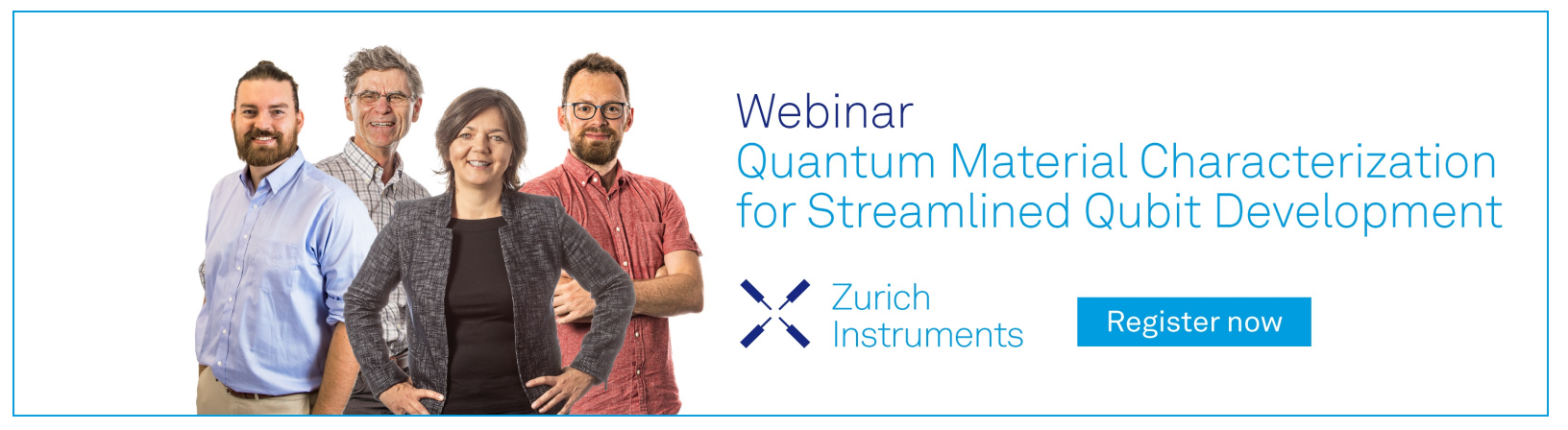




\title{
Room temperature operation of $n$-type Ge/SiGe terahertz quantum cascade lasers predicted by non-equilibrium Green's functions
}

Cite as: Appl. Phys. Lett. 114, 111102 (2019); doi: 10.1063/1.5082172

Submitted: 19 November 2018 • Accepted: 24 February 2019 .

Published Online: 20 March 2019

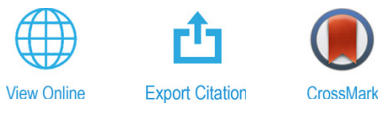

Thomas Grange, ${ }^{1, a)}$ (D) David Stark, ${ }^{2}$ (D) Giacomo Scalari, ${ }^{2}$ (D) Jérôme Faist, ${ }^{2}$ (D) Luca Persichetti, $^{3}$ Luciana Di Gaspare, ${ }^{3}$ (D) Monica De Seta, ${ }^{3}$ (D) Michele Ortolani, ${ }^{4}$ (iD Douglas J. Paul, ${ }^{5}$ (D) Giovanni Capellini, ${ }^{3,6}$ (iD Stefan Birner, ${ }^{7}$ (iD and Michele Virgilio ${ }^{7}$

\author{
AFFILIATIONS \\ 'nextnano $\mathrm{GmbH}$, Garching b. München 85748, Germany \\ ${ }^{2}$ Institute for Quantum Electronics, ETH Zurich, Zurich 8093, Switzerland \\ ${ }^{3}$ Dipartimento di Scienze, Università Roma Tre, Roma 00146, Italy \\ ${ }^{4}$ Dipartimento di Fisica, Università di Roma "La Sapienza," Rome 00185, Italy \\ ${ }^{5}$ School of Engineering, University of Glasgow, Glasgow G12 8LT, United Kingdom \\ ${ }^{6}$ IHP - Leibniz-Institut für innovative Mikroelektronik, Im Technologiepark 25, D-15236 Frankfurt (Oder), Germany \\ ${ }^{7}$ Dipartimento di Fisica “E. Fermi," Università di Pisa, Pisa 56127, Italy \\ a)Electronic mail: thomas.grange@nextnano.com
}

\begin{abstract}
$n$-type Ge/SiGe terahertz quantum cascade lasers are investigated using non-equilibrium Green's functions calculations. We compare the temperature dependence of the terahertz gain properties with an equivalent GaAs/AlGaAs quantum cascade laser design. In the Ge/SiGe case, the gain is found to be much more robust to temperature increase, enabling operation up to room temperature. The better temperature robustness with respect to III-V is attributed to the much weaker interaction with optical phonons. The effect of lower interface quality is investigated and can be partly overcome by engineering smoother quantum confinement.
\end{abstract}

Published under license by AIP Publishing. https://doi.org/10.1063/1.5082172

Terahertz (THz) quantum cascade lasers (QCLs) have been demonstrated with different III-V materials including GaAs/AlGaAs, ${ }^{1}$ InGaAs/AlInAs, ${ }^{2,3}$ InGaAs/GaAsSb, ${ }^{4}$ and InAs/AlAsSb. ${ }^{5}$ In the past decade however, relatively small progress has been reported to increase the maximum operating temperature (presently $200 \mathrm{~K}$ ) despite substantial efforts of design optimization. ${ }^{6-8}$ The rationale for the quenching of $\mathrm{THz}$ laser emission above this temperature is due to the very effective electron-phonon (e-phonon) interaction, typical of III-V materials. Indeed in polar lattices, the longitudinal optical (LO) phonons induce a long-range polarization field which strongly couples to the charge carriers (Fröhlich interaction). The $\mathrm{THz}$ transitions are typically designed to be well below the optical phonon energy (30-36 meV), so that at low temperature, the upper laser state is protected against scattering by emission of LO-phonons. With increasing temperature however, the thermally activated electrons in the subband of the upper lasing state gain enough in-plane kinetic energy to access this scattering channel. ${ }^{9}$ This non-radiative relaxation of carriers reduces the population inversion and is responsible for quenching of the laser emission with increasing temperature as the gain drops below the cavity losses.

To overcome this limitation, QCLs based on crystals having large optical phonon energy such as $\mathrm{GaN}$ or $\mathrm{ZnO}$ have recently been proposed. ${ }^{10}$ As an alternative strategy, non-polar material systems are attractive because of their weaker e-phonon interaction. Indeed in these crystals, the e-phonon coupling is controlled by the deformation potential which due to its short range is much less effective than the Fröhlich interaction. Among different configurations (electron or hole based, Si or Ge rich regimes), ${ }^{11-14}$ theoretical studies have indicated $n$-type $\mathrm{Ge} / \mathrm{SiGe}$ heterostructures where charge transport is associated with L electrons, as the most promising architecture. ${ }^{15-17,41}$ Experimentally, sharp $\mathrm{THz}$ absorption peaks, related to intersubband transitions in $n$-type strain compensated $\mathrm{Ge} / \mathrm{SiGe}$ quantum wells (QWs), have been demonstrated in the $20-50 \mathrm{meV}$ region ${ }^{18-20}$ which interestingly covers the Reststrahlen band of III-V compounds. 
Moreover, long subband lifetimes have been demonstrated, ${ }^{21-24}$ as well as control of carrier tunnelling between coupled QWs. ${ }^{25}$

$\mathrm{THz}$ gain in $n$-type Ge/SiGe QCL structures has been previously predicted using rate equation methods ${ }^{15,16,26}$ and a density matrix formalism. ${ }^{27}$ However, (i) dephasing effects were either not accounted (rate equation models) or described with phenomenological parameters (density matrix), while (ii) the effective electron temperature was entering the models as a free external input parameter. Yet, dephasing in THz QCLs is a crucial issue since linewidths are comparable to the energy separation between the laser levels. In addition, a proper treatment of the transport-induced carrier heating effects is of paramount importance in $\mathrm{Ge} / \mathrm{SiGe}$ systems, due to the low rate of energy transfer from the electronic to the phononic degrees of freedom, associated with the weak e-phonon interaction. In this regard, more predictive calculations can be expected from the non-equilibrium Green's function (NEGF) formalism since (i) it does not require a phenomenological description of dephasing, as all the scattering processes are directly calculated from the material parameters; (ii) there is no need of $a$ priori assumption for the in-plane electron distribution, as carrier heating is accounted for in a self-consistent way.

In this work, to assess the potential of the SiGe alloy material system as a gain medium for intersubband cascade devices, we use the NEGF formalism to benchmark a Ge/SiGe 4-quantum well QCL against a GaAs/AlGaAs counterpart. ${ }^{28}$ To this aim, we preliminary validate our model comparing simulated threshold current densities at different lattice temperatures with experimental data obtained with the GaAs/AlGaAs device, achieving good agreement. Our main findings confirm that $\mathrm{Ge} / \mathrm{SiGe}$ devices, although featuring at low temperature a reduced material gain with respect to III-V systems, are much less sensitive to an increase in temperature. As a consequence, we predict that, by leveraging on efficient waveguides with optical losses not larger than $20 \mathrm{~cm}^{-1}$, room temperature operation can be achieved in multilayer systems with interface roughness (IFR) lower than $2 \AA$.

The NEGF formalism has been shown to provide a powerful framework for investigating vertical transport and gain properties in QCLs. ${ }^{8,29-31}$ Here, we perform NEGF calculations using the nextnano.QCL simulation package which is based on the model described in Refs. 32 and 33 where scattering by acoustic and optical phonons, charged impurities, IFR, and alloy disorders has been accurately modeled by taking into account the full dependence of the scattered inplane momentum. In addition, e-e scattering is included in a selfconsistent one-particle elastic approximation, assuming that the calculated carrier density represents a fixed charge distribution for Coulomb scattering. ${ }^{34}$ This computational efficient approximation is responsible for a reduction of the gain which is similar to the one predicted by the more advanced modeling of e-e scattering reported in Refs. 8 and 35.

For the Ge/SiGe material system with a [001] growth direction, we consider electron transport as due to carriers belonging to the fourfold degenerate L-valleys. Since in the (001) reference system, the L-point mass tensor is not diagonal, confinement and inplane masses are obtained following the transformation introduced by Stern and Howard. ${ }^{15,19,36}$ The interaction with optical phonons in non-polar materials is controlled by the deformation potential only, which in our model is accounted by using the same value of $3.5 \times 10^{8} \mathrm{eV} / \mathrm{cm}$ to describe both intra- and inter-valley events; furthermore, in both the cases, we use an effective dimensionless branch at $37 \mathrm{meV} .^{22}$
To compare the performance of SiGe based THz QCL devices against their III-V counterparts, we choose as a reference the four-well bound-to-continuum design introduced in Ref. 28 using the GaAs/ AlGAs material system. Our choice is motivated by the scalability of this design in terms of emission frequency and by its robustness against deviations of the layer thicknesses or concentrations from the nominal values, which made it very suited for heterogeneous cascade devices. ${ }^{37}$ As shown in Fig. 1(a), carrier injection is based on resonant tunneling from level 1 to level 2; the lasing transition occurs from the latter state to level 3, while levels 4 and 5 act as continuum to extract the carrier from the lower laser level. Finally, relaxation in the injector state involves resonant emission of optical phonons (5-1 of next period). As shown in Fig. 1(b), a very similar electronic spectrum can be engineered in a QCL architecture based on a $\mathrm{Ge} / \mathrm{Si}_{0.23} \mathrm{Ge}_{0.77}(001)$ heterostructure, which features a comparable band-offset, properly adjusting layer thicknesses to account for the heavier confinement $\mathrm{L}$ mass $\left(0.12 m_{0}\right){ }^{15}$ Minimizing the elastic energy associated with the tensile (compressive) SiGe barriers (Ge wells), we find that this system matches the strain-balance conditions when a relaxed $\mathrm{Ge} / \mathrm{Si}_{0.055} \mathrm{Ge}_{0.945}$ virtual substrate is adopted. Note that this strain field splits the sixfold $\Delta$ degeneracy, lowering (rising) the energy of the two valleys located along the growth direction in the barrier (well) region. It follows that $\Delta_{2}$ states well confined in the $\mathrm{Si}_{0.23} \mathrm{Ge}_{0.77}$ layers are present in the same energy region as the relevant L states [see Fig. 1(b)]. We expect that these states play a minor role in the transport process, due to their strong confinement. Moreover, intersubband transitions between $\Delta_{2}$ levels occur at frequencies much larger than the one involving levels 2 and 3. For these reasons, we expect that those states have a limited impact on the gain spectra and therefore we neglect their presence in the NEGF simulations.

Three relevant material parameters differ notably between the GaAs/AlGaAs and Ge/SiGe material systems (see the supplementary material). First, the effective mass along the growth direction is higher

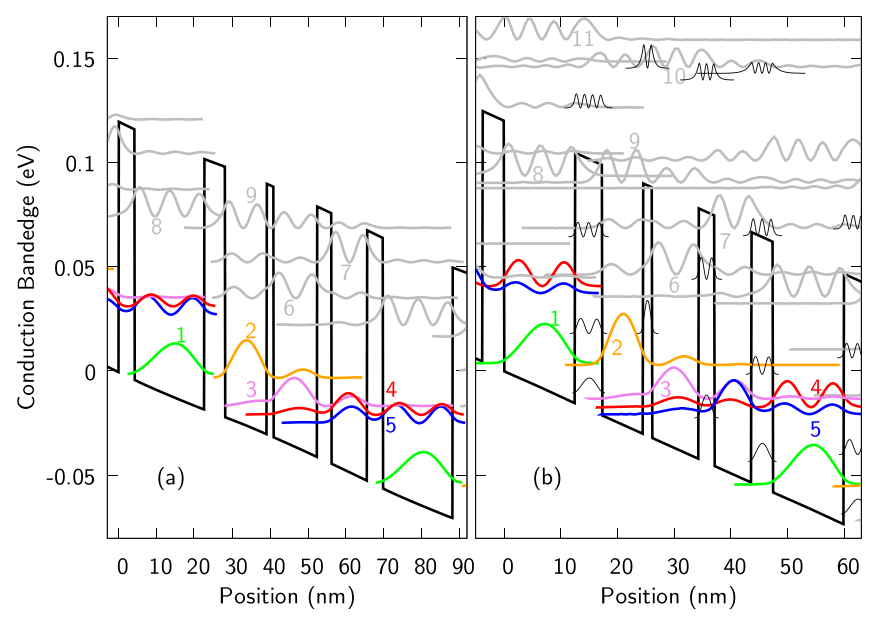

FIG. 1. The conduction band profile and electronic states (squared modulus) for the four-well GaAs/Al ${ }_{0.15} \mathrm{Ga}_{0.85} \mathrm{As}$ (a) and $\mathrm{Ge}_{\mathrm{Si}} \mathrm{Si}_{0.23} \mathrm{Ge}_{0.77}$ (b) $\mathrm{QCL}$ design calculated for the applied electric fields of 7.9 and $12.2 \mathrm{kV} / \mathrm{cm}$, respectively. The electronic states shown are solutions of the Schrödinger equation on a single period (tight-binding basis). In panel (b), $\Delta_{2}$ states confined in the barriers are also shown (dark grey lines). 
in $\mathrm{Ge}(0.12)$ than that in GaAs (0.07), which is detrimental for optical amplification since dipole matrix elements scale as $m^{-1 / 2}$. Second, as already noticed, the interaction with optical phonons is much weaker in $\mathrm{Ge}$ as optical lattice excitations do not induce long-range polarization fields. ${ }^{22}$ Third, the dielectric constant is higher in Ge (16.2) than in GaAs (12.9). This has a favorable consequence, as the elastic scattering rate due to Coulomb interaction (e-impurity and e-e interactions), which has been identified as the principle source of dephasing in GaAs/AlGaAs THz QCLs, ${ }^{33}$ scales as the inverse square of the dielectric constant.

To validate the predictivity of our NEGF approach, we compare in Fig. 2 the calculated threshold current as a function of temperature for the GaAs/AlGaAs device with the experimental data reported in Ref. 28, assuming cavity losses of $25 \mathrm{~cm}^{-1}$, in line with the estimation provided by the authors. A much better agreement is obtained compared to numerical results based on the density matrix model developed in Ref. 28. It is worth noticing that no fitting parameters are used, the IFR scattering playing only a minor role since very similar values for the threshold current are obtained completely neglecting this scattering channel (not shown). For the Ge/SiGe device, assuming the same IFR root-mean square (rms) value of $1 \AA$, we observe a much smaller variation of threshold current densities as a function of the temperature, with an operating range extending up to $300 \mathrm{~K}$ for cavity losses of $20 \mathrm{~cm}^{-1}$.

In Fig. 3, we compare the value of the peak material gain as a function of temperature in the GaAs/AlGaAs and Ge/SiGe QCLs. At low temperatures, the peak gain is higher in the III-V device. In this case however, the gain rapidly drops as the temperature increases. This drop leads to a maximum operation temperature of $168 \mathrm{~K}$, in good agreement with the experimental value of $150 \mathrm{~K}$ reported in Ref. 28. Conversely, the maximum gain for the Ge/SiGe QCL displays a different behavior, being weaker at low temperature but much more robust against the temperature increase. In line with the above results, the predicted gain around $300 \mathrm{~K}$, although reduced to $\gtrsim 20 \mathrm{~cm}^{-1}$,

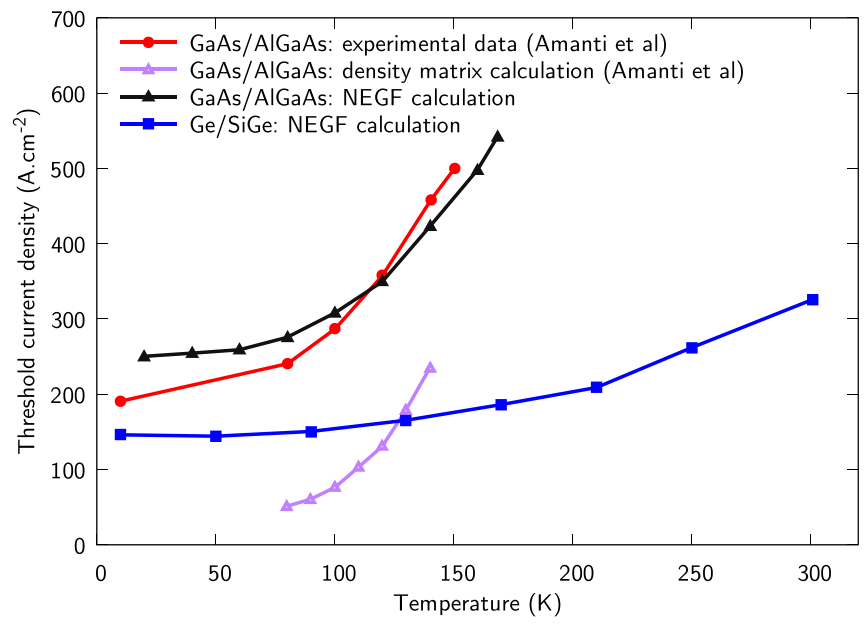

FIG. 2. The threshold current density as a function of lattice temperature for the four-well Ge/SiGe QCL (blue squares) and the GaAs/AIGaAs design. For the III-V device, experimental measurements from Ref. 28 (red circles) are compared with theoretical data based on a density matrix model reported therein (purple open triangles) and with the results of our NEGF model (black filled triangles).

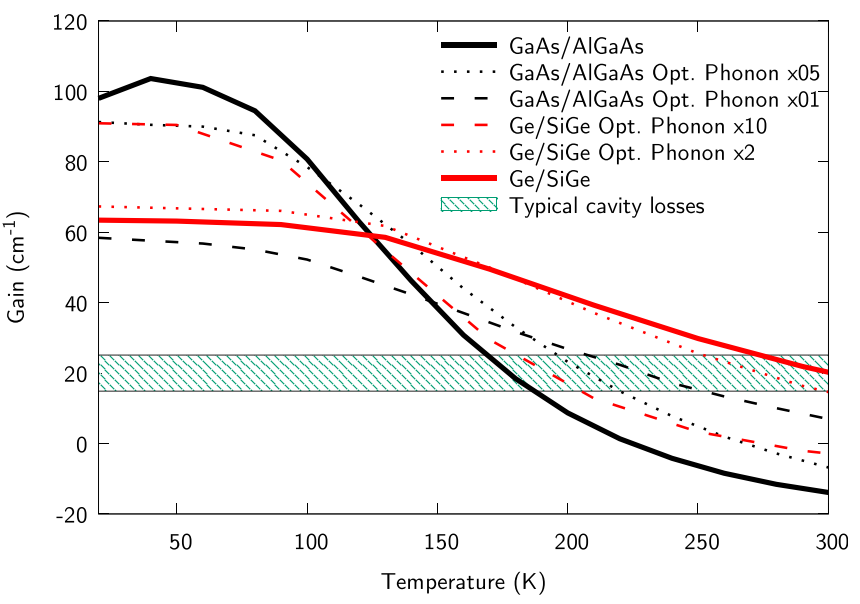

FIG. 3. The maximum gain as a function of temperature calculated for the GaAs/ AIGaAs design of Amanti et al. (black solid line) and the proposed Ge/SiGe design (red solid line). The black dotted and dashed lines have been obtained for the GaAs/AIGaAs QCL scaling the square of the optical-phonon coupling constant by 0.5 and 0.1 , respectively. The red dotted and dashed lines refer to the $\mathrm{Ge} / \mathrm{SiGe}$ QCL with a scaling for the square of the optical-phonon coupling of 2 and 10 , respectively. The horizontal stripe around $20 \mathrm{~cm}^{-1}$ represents typical values for cavity losses.

remains larger than the cavity losses expected in optimized waveguides, thus maintaining the laser emission.

This remarkable difference in the temperature dependence of the two QCL devices can be attributed to the much weaker e-phonon interaction of non-polar lattices, as demonstrated below by artificially tuning the e-optical-phonon coupling constant in the two systems. To this aim, in Fig. 3, we report the peak gain obtained scaling, the square of the e-optical-phonon coupling so as to suppress (enhance) the scattering rate in the GaAs/AlGaAs (Ge/SiGe) QCL. At low temperature, a weaker e-phonon interaction in the III-V based device diminishes the gain but increases it in the high temperature region while the opposite happens upon increasing the interaction in the Ge/SiGe system. This behavior can be understood by the double role played by optical phonons. In fact, for what concerns the lasing transition, increasing the optical-phonon scattering rate is detrimental for the population inversion, especially at high temperature since the scattering from the upper to the lower laser subband can be efficiently activated by the thermal energy. On the other hand, one has to consider that scattering by optical phonons also controls the relaxation from the lower laser level to the injector state of the next period. It follows that when the scattering is reduced, the relaxation rate towards the injector level, which in the design considered is based on optical phonon emission becomes slower and this fact, which is the dominant effect at low temperature, negatively impacts the gain.

In Fig. 4, we compare the position and energy-resolved density of states (top), carrier density (middle), and current density (bottom) calculated for the GaAs/AlGaAs and the Ge/SiGe QCL at the electrical bias corresponding to the peak gain for a lattice temperature of $300 \mathrm{~K}$. As in Fig. 1, a larger number of excited subbands have been considered for the $\mathrm{Ge} / \mathrm{SiGe}$ device in order to account for the larger electronic excess temperature. From the top panels, it is apparent that the electronic spectra in the low energy region are very similar. Nevertheless, 


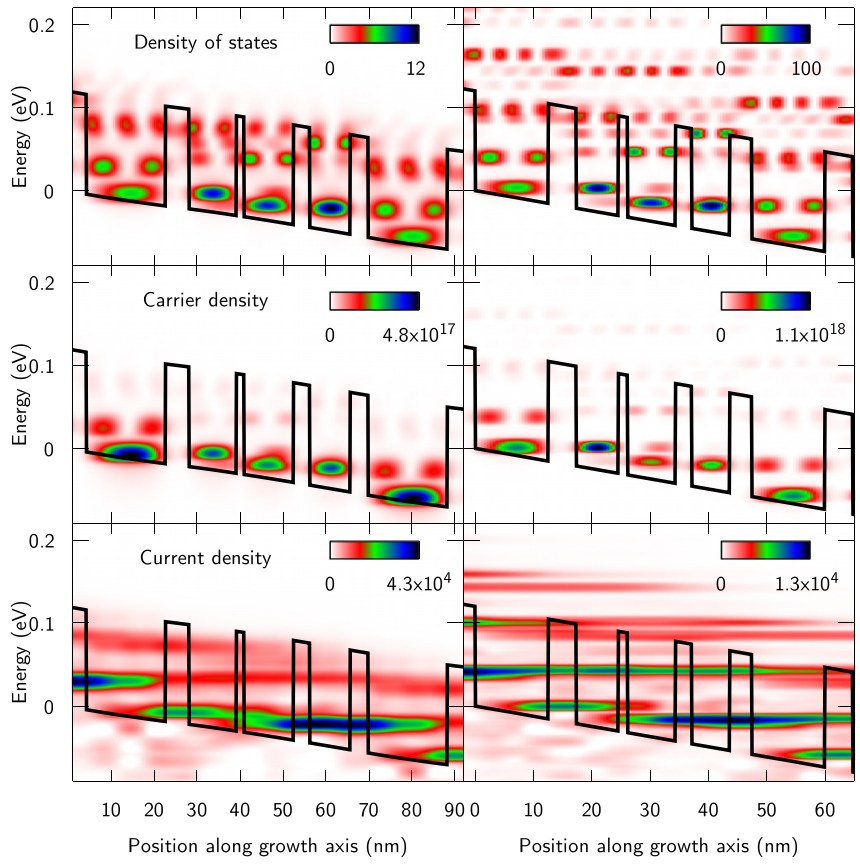

FIG. 4. Position and energy-resolved $1 \mathrm{D}$ density of states (top) in $\left(\mathrm{nm}^{-1} \mathrm{eV}^{-1}\right)$, carrier density (middle) in $\left(\mathrm{cm}^{-3} \mathrm{eV}^{-1}\right)$, and current density (bottom) in $\left(\mathrm{A} \mathrm{cm}^{-2}\right.$ $\mathrm{eV}^{-1}$ ) for the GaAs/AIGaAs design (left) and for the Ge/SiGe design (right) at a lattice temperature of $300 \mathrm{~K}$.

the carrier population of the higher energy levels is negligible in the GaAs/AlGaAs case only (middle panels). This fact has a relevant impact on the current distribution since the higher-energy occupied states contribute significantly to the charge transport as shown in the bottom panels. This effect is related to the much less efficient carrier thermalization in the $\mathrm{Ge} / \mathrm{SiGe}$ device, caused by the weaker interaction with the lattice degrees of freedom. As a consequence, the excess electron effective temperature, evaluated from the in-plane population distribution at room temperature, is found to be much higher $(100 \mathrm{~K})$ than that in the $\mathrm{Ga} / \mathrm{AlGaAs}$ device $(15 \mathrm{~K})$. Another effect related to the large effective temperature is the presence of parasitic absorption peaks associated with thermally activated transitions, involving carriers belonging to the higher energy subbands. In the gain spectrum shown in Fig. 5(a), a dip is calculated at a photon energy of $22 \mathrm{meV}$ owing to the absorption from level 6 to level 7. This absorption feature is not observed in the GaAs/AlGaAs case because of the much smaller population of level 6. In contrast, in the Ge/SiGe case, this parasitic absorption has to be accounted in the design to prevent overlapping with the gain peak. To this aim, we have adopted QWs which are narrow enough to push this absorption line to an energy $(22 \mathrm{meV})$ well above the one of the lasing transition $(16 \mathrm{meV})$. This issue, which has not been considered in previous studies of $\mathrm{Ge} / \mathrm{SiGe}$ QCLs, ${ }^{26}$ should be carefully taken into account when optimizing a Ge/SiGe QCL design.

We now discuss the role played by IFR in the Ge/SiGe system, as the interface quality in $\mathrm{Ge} / \mathrm{SiGe}$ multilayers grown by CVD could be lower than the typical one achievable for GaAs/AlGaAs structures grown by $\mathrm{MBE}{ }^{19,38}$ Indeed, the results presented so far have been obtained assuming the same IFR parameters for GaAs/AlGaAs and Ge/SiGe QCLs, namely, a rms deviation in the growth direction of $1 \AA$ and an in-plane correlation length of $7 \mathrm{~nm}$. To shed light on the role of IFR, in Fig. 5(a), we have calculated the gain spectra with different values of the rms deviation from the ideal interface for the $\mathrm{Ge} / \mathrm{SiGe}$ QCL introduced above (left panel). As the IFR increases, the peak gain decreases at both low and high temperatures. Again, setting cavity losses at $20 \mathrm{~cm}^{-1}$, we find that an IFR rms deviation of $2 \AA$ enables operation only at low temperatures, while a larger value of $3.5 \AA$ prevents any lasing action.

To mitigate the effect of IFR, more advanced designs can be envisaged. ${ }^{39,40}$ We propose in Fig. 5(c) a modified design in which smoother interfaces are engineered by adding layers with a lower $\mathrm{Si}$ concentration $\left(\mathrm{Si}_{0.11} \mathrm{Ge}_{0.89}\right)$. A $1 \mathrm{~nm}$ thick layer of $\mathrm{Si}_{0.11} \mathrm{Ge}_{0.89}$ is

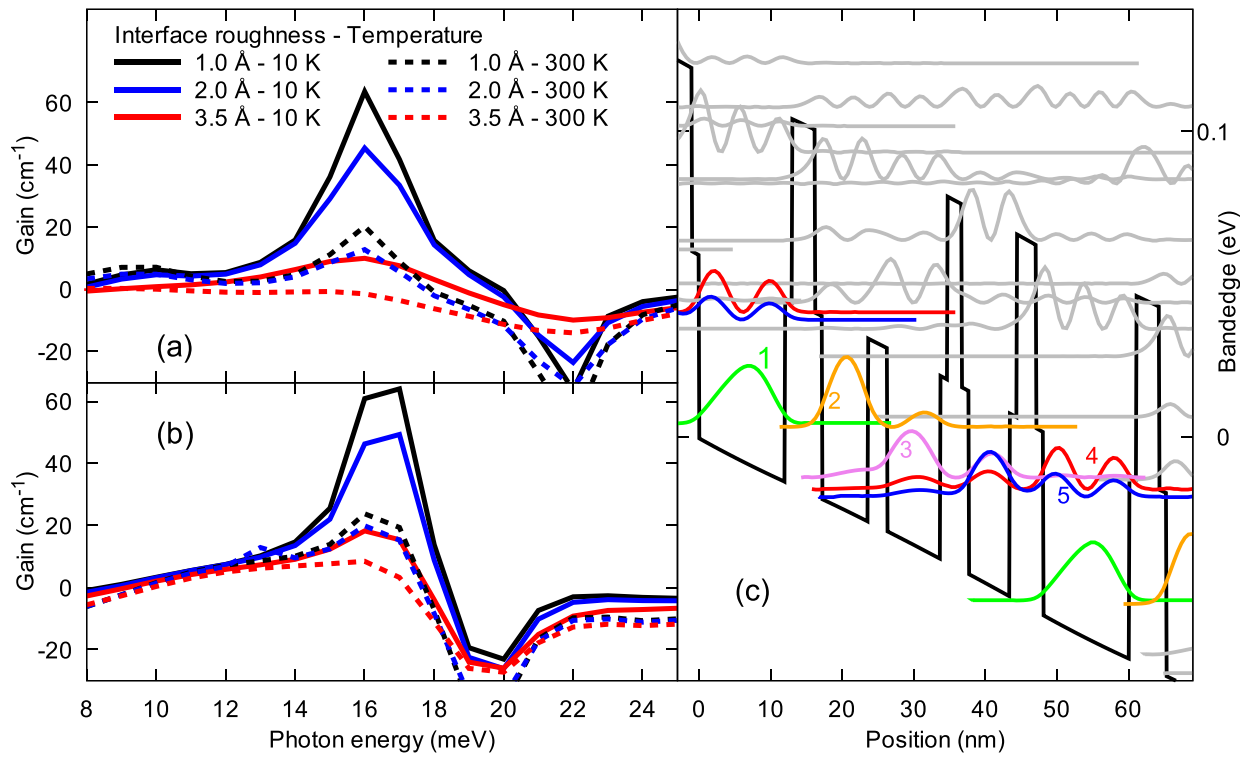

FIG. 5. Material gain spectra for the Ge/ SiGe QCL device with a single barrier height (a) and multiple barrier heights (b) at lattice temperatures of $10 \mathrm{~K}$ (solid lines) and $300 \mathrm{~K}$ (dotted lines) for various interface roughness rms deviations at a constant correlation length $(70 \AA)$. The modified design with multiple barrier heights is displayed in (c). 
introduced at each interface between $\mathrm{Ge}$ and $\mathrm{Si}_{0.23} \mathrm{Ge}_{0.77}$. The barrier separating the two laser levels is replaced by a thicker $\mathrm{Si}_{0.11} \mathrm{Ge}_{0.89}$ barrier. The 5 active levels of the QCLs have energies lower than the intermediate barrier height, and their probability density at the interface with the $\mathrm{Si}_{0.23} \mathrm{Ge}_{0.77}$ barrier material is reduced, owing to the evanescent decay of their wavefunctions in the intermediate barrier layer. In this way, these active levels mainly interact with the IFR contact potential associated with a reduced barrier height of $60 \mathrm{meV}$ instead of $120 \mathrm{meV}$ and then lower IFR scattering rates are expected. Optical gain for this improved design is shown in Fig. 5(b). The spectra are found to be more robust against IFR, confirming that such or similar design strategies can be pursued to overcome the detrimental effects associated with a large IFR.

In summary, using NEGF simulations, we have assessed the potential of the $\mathrm{Ge} / \mathrm{SiGe}$ material system to achieve $\mathrm{THz}$ emission at room temperature in QCL devices through a detailed comparison with an equivalent GaAs/AlGaAs design. The Ge/SiGe QCL is found to be significantly more robust as the temperature increases, which is clearly attributed to the weaker electron-phonon interaction. Finally, we have shown that detrimental effects related to possibly high IFR can be attenuated by engineering a smoother confinement profile adopting a three layer barrier. We believe that the present results will motivate new experimental efforts aimed at demonstrating room-temperature operation in group IV QCL THz devices.

See supplementary material for the relevant material parameters used in the simulation of $\mathrm{GaAs} / \mathrm{AlGaAs}$ and $\mathrm{Ge} / \mathrm{SiGe}$ systems.

This project was funded by the European Union's Horizon 2020 research and innovation programme under Grant Agreement No. 766719 (FLASH).

\section{REFERENCES}

${ }^{1}$ R. Köhler, A. Tredicucci, F. Beltram, H. E. Beere, E. H. Linfield, A. G. Davies, D. A. Ritchie, R. C. Iotti, and F. Rossi, Nature 417, 156 (2002).

${ }^{2}$ L. Ajili, G. Scalari, N. Hoyler, M. Giovannini, and J. Faist, Appl. Phys. Lett. 87, 141107 (2005).

${ }^{3}$ C. Deutsch, A. Benz, H. Detz, P. Klang, M. Nobile, A. M. Andrews, W. Schrenk, T. Kubis, P. Vogl, G. Strasser et al., Appl. Phys. Lett. 97, 261110 (2010).

${ }^{4}$ C. Deutsch, M. Krall, M. Brandstetter, H. Detz, A. Andrews, P. Klang, W. Schrenk, G. Strasser, and K. Unterrainer, Appl. Phys. Lett. 101, 211117 (2012).

${ }^{5}$ M. Brandstetter, M. A. Kainz, T. Zederbauer, M. Krall, S. Schönhuber, H. Detz, W. Schrenk, A. M. Andrews, G. Strasser, and K. Unterrainer, Appl. Phys. Lett. 108, 011109 (2016).

${ }^{6}$ S. Kumar, Q. Hu, and J. L. Reno, Appl. Phys. Lett. 94, 131105 (2009).

${ }^{7}$ S. Fathololoumi, E. Dupont, C. Chan, Z. Wasilewski, S. Laframboise, D. Ban, A. Mátyás, C. Jirauschek, Q. Hu, and H. Liu, Opt. Express 20, 3866 (2012).

${ }^{8}$ M. Franckié, L. Bosco, M. Beck, C. Bonzon, E. Mavrona, G. Scalari, A. Wacker, and J. Faist, Appl. Phys. Lett. 112, 021104 (2018).

${ }^{9}$ B. S. Williams, Nat. Photonics 1, 517 (2007).
${ }^{10}$ K. Wang, T. Grange, T.-T. Lin, L. Wang, Z. Jéhn, S. Birner, J. Yun, W. Terashima, and H. Hirayama, Appl. Phys. Lett. 113, 061109 (2018).

${ }^{11}$ L. Friedman, G. Sun, and R. A. Soref, Appl. Phys. Lett. 78, 401 (2001).

${ }^{12}$ S. A. Lynch, R. Bates, D. Paul, D. Norris, A. Cullis, Z. Ikonic, R. Kelsall, P. Harrison, D. Arnone, and C. Pidgeon, Appl. Phys. Lett. 81, 1543 (2002).

${ }^{13}$ L. Lever, A. Valavanis, Z. Ikonić, and R. Kelsall, Appl. Phys. Lett. 92, 021124 (2008).

${ }^{14}$ G. Matmon, D. Paul, L. Lever, M. Califano, Z. Ikonić, R. Kelsall, J. Zhang, D. Chrastina, G. Isella, H. Von Känel et al., J. Appl. Phys. 107, 053109 (2010).

${ }^{15}$ K. Driscoll and R. Paiella, J. Appl. Phys. 102, 093103 (2007).

${ }^{16}$ A. Valavanis, L. Lever, C. Evans, Z. Ikonić, and R. Kelsall, Phys. Rev. B 78, 035420 (2008).

${ }^{17}$ A. Valavanis, T. Dinh, L. Lever, Z. Ikonić, and R. Kelsall, Phys. Rev. B 83, 195321 (2011).

${ }^{18}$ M. De Seta, G. Capellini, Y. Busby, F. Evangelisti, M. Ortolani, M. Virgilio, G. Grosso, G. Pizzi, A. Nucara, and S. Lupi, Appl. Phys. Lett. 95, 051918 (2009).

${ }^{19}$ Y. Busby, M. De Seta, G. Capellini, F. Evangelisti, M. Ortolani, M. Virgilio, G. Grosso, G. Pizzi, P. Calvani, S. Lupi et al., Phys. Rev. B 82, 205317 (2010).

${ }^{20}$ M. De Seta, G. Capellini, M. Ortolani, M. Virgilio, G. Grosso, G. Nicotra, and P. Zaumseil, Nanotechnology 23, 465708 (2012).

${ }^{21}$ M. Ortolani, D. Stehr, M. Wagner, M. Helm, G. Pizzi, M. Virgilio, G. Grosso, G. Capellini, and M. De Seta, Appl. Phys. Lett. 99, 201101 (2011).

${ }^{22}$ M. Virgilio, M. Ortolani, M. Teich, S. Winnerl, M. Helm, D. Sabbagh, G. Capellini, and M. De Seta, Phys. Rev. B 89, 045311 (2014).

${ }^{23}$ D. Sabbagh, J. Schmidt, S. Winnerl, M. Helm, L. Di Gaspare, M. De Seta, M. Virgilio, and M. Ortolani, ACS Photonics 3, 403 (2016).

${ }^{24}$ M. Virgilio, G. Grosso, G. Pizzi, M. De Seta, G. Capellini, and M. Ortolani, Phys. Rev. B 86, 205317 (2012).

${ }^{25}$ C. Ciano, M. Virgilio, M. Montanari, L. Persichetti, L. D. Gaspare, M. Ortolani, L. Baldassarre, M. H. Zoellner, O. Skibitzki, G. Scalari, J. Faist, M. S. D. J. Paul, G. Nicotra, S. B. T. Grange, G. Capellini, and M. D. Seta, Phys. Rev. Appl. 11, 014003 (2019).

${ }^{26}$ L. Lever, A. Valavanis, C. Evans, Z. Ikonić, and R. Kelsall, Appl. Phys. Lett. 95, 131103 (2009).

${ }^{27}$ T. Dinh, A. Valavanis, L. Lever, Z. Ikonić, and R. Kelsall, Phys. Rev. B 85, 235427 (2012).

${ }^{28}$ M. I. Amanti, G. Scalari, R. Terazzi, M. Fischer, M. Beck, J. Faist, A. Rudra, P. Gallo, and E. Kapon, New J. Phys. 11, 125022 (2009).

${ }^{29}$ S.-C. Lee and A. Wacker, Phys. Rev. B 66, 245314 (2002).

${ }^{30}$ R. Nelander and A. Wacker, Appl. Phys. Lett. 92, 081102 (2008).

${ }^{31}$ T. Kubis, C. Yeh, P. Vogl, A. Benz, G. Fasching, and C. Deutsch, Phys. Rev. B 79, 195323 (2009).

${ }^{32}$ T. Grange, Phys. Rev. B 89, 165310 (2014).

${ }^{33}$ T. Grange, Phys. Rev. B 92, 241306 (2015).

${ }^{34}$ T.-T. Lin, L. Wang, K. Wang, T. Grange, and H. Hirayama, Appl. Phys. Express 11, 112702 (2018).

${ }^{35}$ D. O. Winge, M. Franckié, C. Verdozzi, A. Wacker, and M. F. Pereira, J. Phys.: Conf. Ser. 696, 012013 (2016).

${ }^{36}$ F. Stern and W. E. Howard, Phys. Rev. 163, 816 (1967).

${ }^{37}$ M. Rösch, G. Scalari, M. Beck, and J. Faist, Nat. Photonics 9, 42 (2015).

${ }^{38}$ M. Califano, N. Vinh, P. Phillips, Z. Ikonić, R. Kelsall, P. Harrison, C. Pidgeon, B. Murdin, D. Paul, P. Townsend et al., Phys. Rev. B 75, 045338 (2007).

${ }^{39}$ Y. Bai, N. Bandyopadhyay, S. Tsao, E. Selcuk, S. Slivken, and M. Razeghi, Appl. Phys. Lett. 97, 251104 (2010).

${ }^{40}$ M. Semtsiv, Y. Flores, M. Chashnikova, G. Monastyrskyi, and W. Masselink, Appl. Phys. Lett. 100, 163502 (2012).

${ }^{41}$ G. Sun, H. H. Cheng, and J. Menendez, Appl. Phys. Lett. 90, 251105 (2007). 